\title{
Probabilistic Representations for the Solution of Higher Order Differential Equations
}

\author{
S. Mazzucchi ${ }^{1,2}$ \\ ${ }^{1}$ CIRM Centro Internazionale per la Ricerca Matematica, Fondazione Bruno Kessler, 38123 Trento, Italy \\ ${ }^{2}$ Dipartimento di Matematica, Università di Trento, Via Sommarive 14, 38123 Trento, Italy \\ Correspondence should be addressed to S. Mazzucchi; mazzucch@science.unitn.it
}

Received 16 April 2013; Revised 12 June 2013; Accepted 13 June 2013

Academic Editor: David Holcman

Copyright (c) 2013 S. Mazzucchi. This is an open access article distributed under the Creative Commons Attribution License, which permits unrestricted use, distribution, and reproduction in any medium, provided the original work is properly cited.

A probabilistic representation for the solution of the partial differential equation $(\partial / \partial t) u(t, x)=-\alpha \Delta^{2} u(t, x), \alpha \in \mathbb{C}$, is constructed in terms of the expectation with respect to the measure associated to a complex-valued stochastic process.

\section{Introduction}

The connection between the solution of parabolic equations associated to second-order elliptic operators and the theory of stochastic processes is a largely studied topic [1]. The main instance is the Feynman-Kac formula, providing a representation of the solution of the heat equation with potential $V \in C_{\infty}\left(\mathbb{R}^{d}\right)$ (the continuous functions vanishing at infinity):

$$
\begin{aligned}
\frac{\partial}{\partial t} u(t, x) & =\frac{1}{2} \Delta u(t, x)-V(x) u(t, x), \quad t \in \mathbb{R}^{+}, x \in \mathbb{R}^{d}, \\
u(0, x) & =u_{0}(x)
\end{aligned}
$$

in terms of an integral with respect to the measure of the Wiener process, the mathematical model of the Brownian motion [2]:

$$
u(t, x)=\int_{C_{t}} e^{-\int_{0}^{t} V(\omega(s)+x) d s} u_{0}(\omega(t)+x) d W(\omega) .
$$

If the Laplacian in (1) is replaced with a higher order differential operator, that is, if we consider a Cauchy problem of the form

$$
\begin{array}{rl}
\frac{\partial}{\partial t} u(t, x) & =(-1)^{N+1} \Delta^{N} u(t, x)-V(x) u(t, x), \\
t & t \in \mathbb{R}^{+}, x \in \mathbb{R}^{d}, \\
u(0, x) & =u_{0}(x),
\end{array}
$$

with $N \in \mathbb{N}, N \geq 2$, then a formula analogous to (2), giving the solution of (3) in terms of the expectation with respect to the measure associated to a Markov process, is lacking. In fact, such a formula cannot be proved for semigroups whose generator does not satisfy the maximum principle, as in the case of $\Delta^{N}$ with $N>1$. In fact in the case where $N>1$ the Cauchy problem (3) is not well posed on the space of continuous bounded functions [3]. In other words it is not possible to find a stochastic process which plays for the parabolic equation (3) the same role that the Wiener process plays for the heat equation.

We would like to point out that the problem of the probabilistic representation of the solution of the Cauchy problem (3) presents some similarities with the problem of the mathematical definition of Feynman path integrals (see [4-7] for a discussion of this topic). Indeed in both cases it is not possible to implement an integration theory of Lebesgue type in terms of a bounded variation measure on a space of continuous paths [8]. An analogous of the Feynman-Kac formula for the parabolic equation (3), namely, an equation of the form

$$
u(t, x)=\int_{\omega(0)=x} e^{-\int_{0}^{t} V(\omega(s)) d s} u_{0}(\omega(t)) d P(\omega)
$$

(where $P$ should be some "measure" on a space of "paths" $\omega$ : $[0, t] \rightarrow \mathbb{R})$, can be obtained only under some restrictions on $u_{0}$ and $V$ and by giving up a traditional integration theory 
in the Lebesgue sense with respect to a bounded variation measure on a space of (real) continuous paths.

In the mathematical literature two main approaches have been proposed. The first one $[9,10]$ realizes formula (4) in terms of the expectation with respect to a signed measure $P_{2 N}$ on a space of paths on the interval $[0, t]$.

It is worthwhile to mention that an analogous of the arcsine law $[10,11]$, of the central limit theorem [12], and of Ito formula and Ito stochastic calculus $[10,13]$ have been proved for the (finite additive) signed measure $P_{2 N}$. Moreover, a Feynman-Kac formula has been proved [9-11], for the representation of the solution of the Cauchy problem (3) in the case where $V$ is a bounded piecewise continuous function and for an initial datum $u_{0} \in C^{2 N}$, by realizing formula (4) as limit of finite dimensional cylindrical approximations [14]. We also mention the work by Levin and Lyons [15] on rough paths, conjecturing that the signed measure $P_{2 N}$ could exist on the quotient space of equivalence classes of paths corresponding to different parametrization of the same path.

A different approach is based on the construction of a stochastic process on a space of complex paths. In this case the integration is performed with respect to a well-defined positive probability measure on a complex space. One of the first results was given by Funaki [16], who constructed a complex stochastic process $\left\{X_{t}\right\}_{t \geq 0}$ by composing two independent Brownian motions $\{B(t)\}_{t \geq 0}$ and $\{w(t)\}_{t \geq 0}$ in the following way:

$$
X_{t}:= \begin{cases}B(w(t)) & \text { if } w(t) \geq 0 \\ i B(-w(t)) & \text { if } w(t)<0\end{cases}
$$

and proving that, for a suitable class of analytic initial datum $u_{0}$, the solution of the Cauchy problem

$$
\begin{aligned}
\frac{\partial u}{\partial t} & =\frac{1}{8} \frac{\partial^{4} u}{\partial x^{4}} \quad-\infty<x<\infty, 0<t<\infty, \\
u(0, x) & =u_{0}(x)
\end{aligned}
$$

is given by the expectation

$$
u(t, x)=\mathbb{E}\left[u_{0}\left(x+X_{t}\right)\right] .
$$

In fact this result can be generalized to partial differential equations of order $2^{n}$, by multiple iterations of suitable processes [16-18]. These results are also related to Bochner subordination [19].

There are also similarities between the Funaki's process $\left\{X_{t}\right\}$ and the "iterated Brownian motion" [20], but the latter is not connected to the probabilistic representation of the solution of a partial differential equation with regular coefficients. In fact the processes constructed by iterating copies of independent BMs (or other process) are associated to higher order PDE of particular form, where the initial datum plays a particular role and enters also in the differential equation [21].

Complex-valued processes, connected to PDE of the form (3), have been also proposed by other authors by means of different techniques. In [22-24] Madrecki et al. consider the fourth degree heat-type equation (6) and construct a probabilistic representation for its solution in terms of a stable probabilistic Borel measure $m$ on the space $\Omega=$ $C\left([0, t], \mathbb{C}^{\infty}\right)$ of continuous mappings on $[0, t]$ with values in the set $\mathbb{C}^{\infty}$ of complex-valued sequences, endowed with the product topology. In this setting a Feynman-Kac type formula is proved, for the fourth order heat equation with linear potential.

Another probabilistic approach is presented by Sainty in [25], where a representation for the solution of $(\partial / \partial t) u(t, x)=$ $\left(\partial^{n} / \partial x^{n}\right) u(t, x)$ is given in terms of the expectation with respect to a particular complex-valued process $X_{[n]}(t), t \geq$ 0 , called "Brownian motion of order $n$ ". It is worthwhile also to mention a completely different approach proposed by Léandre [26, 27], which has some analogies with the mathematical realization of Feynman path integrals by means of white noise calculus [28]. Indeed Léandre has recently constructed a "probabilistic representation" of the solution of the Cauchy problem (3) in terms of an infinite dimensional distribution on the Connes space $[27,29,30]$. Another interesting approach related to the theory of pseudoprocesses introduced by Daletsky and Fomin [31] has been recently proposed by Smorodina and Faddeev [32].

We eventually mention an interesting probabilistic approach to the equation $\Delta^{k} u=0$ described in [33].

The present paper presents the construction of an alternative complex-valued stochastic process generalizing Funaki's result [16] and a corresponding probabilistic representation for the solution of the Cauchy problem:

$$
\begin{aligned}
\frac{\partial u}{\partial t}(x, t)=-\frac{\alpha}{8} & \frac{\partial^{4}}{\partial x^{4}} u(x, t)+V(t, x) u(t, x) \\
& -\infty<x<\infty, 0 \leq t<\infty, \\
u(x, 0)= & u_{0}(x),
\end{aligned}
$$

with $\alpha \in \mathbb{C}$ and for $V, u_{0}$ satisfying suitable analyticity assumptions.

The paper is organized as follows. Section 2 presents the construction of a complex random variable $z_{t}^{\alpha}$ and the representation of the solution of (8) with $V \equiv 0$ in terms of the expectation with respect to the probability measure associated with $z_{t}^{\alpha}$. Section 3 presents the proof of a FeynmanKac type formula for the solution of (8) in the cases where $V$ is linear in the space variables and presents an explicit time dependence.

\section{A Complex-Valued Random Variable Associated to the 4-Order Heat-Type Equation}

In the present section we construct a probabilistic representation for the solution of (8) in the case where $V=0$, namely,

$$
\frac{\partial u}{\partial t}(x, t)=-\frac{\alpha}{8} \frac{\partial^{4} u}{\partial x^{4}}(x, t) \quad-\infty<x<\infty, 0 \leq t<\infty .
$$

An equation of this form in the case where $\alpha \in \mathbb{R}$, as mentioned in the introduction, has been studied by several 
authors by means of different techniques $[9,10,16,25]$. In this section we show that the results in $[9,10,16]$ can be seen as particular cases of a general theory presented in [34-37], connecting the solution of parabolic problems with the solution of related hyperbolic problems.

Given a Banach space $X$ and a strongly continuous group of operators $\left\{T_{A}(t)\right\}_{t \in \mathbb{R}}$ on $X$ with generator $A$, it is possible to construct the holomorphic semigroup $e^{t\left(A^{2} / 2\right)}$ with generator $A^{2} / 2$ in terms of a Gaussian expectation of the group $T(t)$ :

$$
\begin{aligned}
e^{t\left(A^{2} / 2\right)} f & =\mathbb{E}_{N(0, t)}[T(s) f] \\
& =(2 \pi t)^{-1 / 2} \int_{-\infty}^{\infty} e^{-s^{2} / 2 t} T(s) f d s, \quad f \in X .
\end{aligned}
$$

More generally, given a polynomial $P(A)$ in $A$ with complex coefficients, whose leading term has the form $c_{2 m} A^{2 m}$, with $(-1)^{m+1} \operatorname{Re}\left(c_{2 m}\right)>0$, then $P(A)$ generates a holomorphic semigroup on $X$. Its action on a vector $f \in X$ belonging to the domain of $A^{2 m}$ is given by

$$
e^{t P(A)} f=\int_{-\infty}^{\infty} \widehat{g}_{t}(s) T_{A}(s) f d s, \quad f \in X,
$$

with $\widehat{g}_{t}(s)=(1 / 2 \pi) \int_{-\infty}^{\infty} e^{-i s \xi} e^{t P(i \xi)} d \xi$ (see [36]). Equation (11) can be regarded as a particular example of the "method of transmutation" described in [38], which allows to relate different classes of problems by means of a suitable transformation technique.

Let us consider (11) in the case where $X$ is the Hilbert space $L^{2}(\mathbb{R}), A=i(\Delta / 2)$, and $P(x)=x^{2} / 2$. One gets the following representation:

$$
e^{-(t / 8) \Delta^{2}}=\int_{-\infty}^{\infty} e^{i s(\Delta / 2)} \frac{e^{-s^{2} / 2 t}}{\sqrt{2 \pi t}} d s,
$$

giving the semigroup $e^{-(t / 8) \Delta^{2}}$ in terms of a Gaussian expectation, with respect to the time variable $s \in \mathbb{R}$, of the Schrödinger group $e^{i s(\Delta / 2)}$. By applying (12) to an initial datum $u_{0} \in S(\mathbb{R})$ one can write the following chain of equalities:

$$
\begin{aligned}
e^{-(t / 8) \Delta^{2}} u_{0}(x) & \\
= & \int_{-\infty}^{\infty} \frac{e^{-s^{2} / 2 t}}{\sqrt{2 \pi t}} e^{i s(\Delta / 2)} u_{0}(x) d s \\
= & \int_{0}^{\infty} \frac{e^{-s^{2} / 2 t}}{\sqrt{2 \pi t}}\left(e^{i s(\Delta / 2)} u_{0}(x)+e^{-i s(\Delta / 2)} u_{0}(x)\right) d s \\
= & \int_{0}^{\infty} \frac{e^{-s^{2} / 2 t}}{\sqrt{2 \pi t}} \int_{\mathbb{R}}\left(\frac{e^{i\left(y^{2} / 2 s\right)}}{\sqrt{2 \pi i s}} u_{0}(x+y)\right. \\
& \left.+\frac{e^{-i\left(y^{2} / 2 s\right)}}{\sqrt{-2 \pi i s}} u_{0}(x+y)\right) d y d s .
\end{aligned}
$$

Under the assumptions that $u_{0}$ can be extended to an entire function on the complex plane $\mathbb{C}$, denoted again with $u_{0}$, such that for any $h \in \mathbb{R}^{+}$one has that $e^{-h|z|^{2}}\left|u_{0}(z)\right|$ is a bounded function on $\mathbb{C}$, a rotation of the integration path in the complex $y$-plane gives

$$
\begin{aligned}
e^{-(t / 8) \Delta^{2}} u_{0}(x) & \\
=\int_{0}^{\infty} \frac{e^{-s^{2} / 2 t}}{\sqrt{2 \pi t}} \int_{\mathbb{R}} \frac{e^{-y^{2} / 2 s}}{\sqrt{2 \pi s}}\left(\begin{array}{c}
u_{0}\left(x+e^{i \pi / 4} y\right) \\
+
\end{array}\right. & \left.u_{0}\left(x+e^{-i \pi / 4} y\right)\right) d y d s .
\end{aligned}
$$

Analogously, if $\alpha$ is a real positive constant, a change of variable in the latter integral gives

$$
\begin{aligned}
& e^{-(\alpha t / 8) \Delta^{2}} u_{0}(x) \\
&=\int_{0}^{\infty} \frac{e^{-s^{2} / 2 t}}{\sqrt{2 \pi t}} \int_{\mathbb{R}} \frac{e^{-y^{2} / 2 s}}{\sqrt{2 \pi s}}\left(u_{0}\left(x+\alpha^{1 / 4} e^{i \pi / 4} y\right)\right. \\
&\left.+u_{0}\left(x+\alpha^{1 / 4} e^{-i \pi / 4} y\right)\right) d y d s .
\end{aligned}
$$

Now the integral on the right hand side of (15) makes sense for a class of functions $u_{0}$ larger than those considered so far, as well as for complex values of the parameter $\alpha$. Let us consider the Cauchy problem

$$
\begin{aligned}
\frac{\partial u}{\partial t}(x, t) & =-\frac{\alpha}{8} \frac{\partial^{4} u}{\partial x^{4}}(x, t) \quad-\infty<x<\infty, 0 \leq t<\infty, \\
u(x, 0) & =u_{0}(x),
\end{aligned}
$$

with $\alpha \in \mathbb{C}$. The next theorems give a characterization of some classes of functions $u_{0}$ such that the integral

$$
\begin{aligned}
\int_{0}^{\infty} \frac{e^{-s^{2} / 2 t}}{\sqrt{2 \pi t}} \int_{\mathbb{R}} \frac{e^{-y^{2} / 2 s}}{\sqrt{2 \pi s}}\left(u_{0}\left(x+\alpha^{1 / 4} e^{i \pi / 4} y\right)\right. \\
\left.+u_{0}\left(x+\alpha^{1 / 4} e^{-i \pi / 4} y\right)\right) d y d s
\end{aligned}
$$

is absolutely convergent and provides a representation for the solution of the Cauchy problem (16). The following theorem is a generalization of Funaki's result [16] to the case where $\alpha$ is an arbitrary complex parameter.

Theorem 1. Let $u_{0}$ be a real valued function satisfying the following properties:

(1) $u_{0}$ can be extended to an entire function on the complex plane $\mathbb{C}$, denoted again with $u_{0}$;

(2) for any $h \in \mathbb{R}^{+}$, one has that $e^{-h|z|^{2}}\left|u_{0}(z)\right|$, $e^{-h|z|^{2}}\left|\partial u_{0}(z) / \partial x\right|$, and $e^{-h|z|^{2}}\left|\partial u_{0}(z) / \partial y\right|$, with $z=x+$ $i y$, are bounded functions on $\mathbb{C}$.

Then the solution of (16) is given by (17). 
Proof. By the assumption that $e^{-h|z|^{2}}\left|u_{0}(z)\right|$ is a bounded functions on $\mathbb{C}$ for any $h \in \mathbb{R}^{+}$, one can easily verify that the integral

$$
\begin{aligned}
u(t, x)=\int_{0}^{\infty} \frac{e^{-s^{2} / 2 t}}{\sqrt{2 \pi t}} \int_{\mathbb{R}} \frac{e^{-y^{2} / 2 s}}{\sqrt{2 \pi s}}( & u_{0}\left(x+\alpha^{1 / 4} e^{i \pi / 4} y\right) \\
& \left.+u_{0}\left(x+\alpha^{1 / 4} e^{-i \pi / 4} y\right)\right) d y d s
\end{aligned}
$$

is absolutely convergent. By estimates

$$
\begin{aligned}
& e^{-h|z|^{2}}\left|\frac{\partial u_{0}(z)}{\partial x}\right|<C, \\
& e^{-h|z|^{2}}\left|\frac{\partial u_{0}(z)}{\partial y}\right|<C
\end{aligned}
$$

and an integration by parts formula, one has that $\left(\partial^{4} / \partial x^{4}\right) u(t$, $x$ ) is given by

$$
\begin{aligned}
& \frac{\partial^{4}}{\partial x^{4}} u(t, x) \\
& =-\frac{8}{\alpha} \int_{0}^{\infty} \frac{e^{-s^{2} / 2 t}}{\sqrt{2 \pi t}}\left(\frac{s^{2}}{2 t^{4}}-\frac{1}{2 t}\right) \\
& \quad \times \int_{\mathbb{R}} \frac{e^{-y^{2} / 2 s}}{\sqrt{2 \pi s}}\left(u_{0}\left(x+\alpha^{1 / 4} e^{i \pi / 4} y\right)\right. \\
& \left.\quad+u_{0}\left(x+\alpha^{1 / 4} e^{-i \pi / 4} y\right)\right) d y d s
\end{aligned}
$$

which is equal to $-(8 / \alpha)(\partial / \partial t) u(t, x)$.

Remark 2. The functions $u_{0}$ satisfying the assumptions (1) and (2) of Theorem 1 include, for instance, polynomials of arbitrary degree, as well as the functions $u_{0}$ that are Fourier transform of measures, that is, of the form

$$
u_{0}(x)=\int_{\mathbb{R}} e^{i k x} d \mu(k),
$$

where $\mu$ is a complex bounded variation measure such that $\forall x, y \in \mathbb{R}:$

(i) $\int_{\mathbb{R}} e^{k y} d|\mu|(k)<\infty, \int_{\mathbb{R}} e^{k y} d|\mu|(k)<\infty,|\mu|$ being the total variation measure of $\mu$,

(ii) for any $h \in \mathbb{R}^{+}$, the functions $(x, y) \mapsto$ $e^{-h\left(x^{2}+y^{2}\right)}\left|\int_{\mathbb{R}} e^{i k x-k y} d \mu(k)\right| \quad$ and $(x, y) \quad \mapsto$ $e^{-h\left(x^{2}+y^{2}\right)}\left|\int_{\mathbb{R}} e^{i k x-k y} d \mu(k)\right|$ are bounded on $\mathbb{R}^{2}$.

Remark 3. The Funaki formula (7) for the solution of (16) in the case where $\alpha=-1$ can be written in the following form:

$$
u(t, x)=\int_{0}^{\infty} \frac{e^{-y^{2} / 2 t}}{\sqrt{2 \pi t}} \int_{\mathbb{R}} \frac{e^{-z^{2} / 2 y}}{\sqrt{2 \pi y}}\left(u_{0}(x+z)+u_{0}(x+i z)\right) d z d y
$$

and can be obtained as a special case of (17).
The following theorem describes a particular class of functions $u_{0}$ such that the integral (17) is absolutely convergent and provides a representation for the solution of the Cauchy problem (16).

Theorem 4. Let $u_{0}$ be a real valued function of the form

$$
u_{0}(x)=\int_{\mathbb{R}} e^{i k x} d \mu(k), \quad x \in \mathbb{R}
$$

where $\mu$ is a complex bounded variation measure on $\mathbb{R}$ satisfying the following bound:

$$
\int_{\mathbb{R}} e^{(|\alpha| t / 8) y^{4}} d|\mu|(y)<\infty
$$

$|\mu|$ being the total variation measure of $\mu$.

Then the solution of the Cauchy problem (16) is given by (17).

Proof. Under the stated assumption on $u_{0}$, the integral (17) assumes the following form:

$$
\begin{array}{r}
\int_{0}^{\infty} \frac{e^{-s^{2} / 2 t}}{\sqrt{2 \pi t}} \int_{\mathbb{R}} \frac{e^{-y^{2} / 2 s}}{\sqrt{2 \pi s}} \int_{\mathbb{R}}\left(e^{i k\left(x+\alpha^{1 / 4} e^{i \pi / 4} y\right)}\right. \\
\left.+e^{i k\left(x+\alpha^{1 / 4} e^{-i \pi / 4} y\right)}\right) d \mu(k)
\end{array}
$$

by Fubini theorem, which holds because of condition (24); the latter is equal to

$$
\int_{\mathbb{R}} e^{i k x} e^{\alpha t y^{4} / 8} d \mu(y)
$$

which is the solution of the Cauchy problem (16).

Equation (17) can be written in terms of the expectation with respect to the measure associated to a complex random variable $z_{t}^{\alpha}$ :

$$
e^{-\alpha(t / 8) \Delta^{2}} u_{0}(x)=\mathbb{E}\left[u_{0}\left(x+z_{t}^{\alpha}\right)\right],
$$

where $z_{t}^{\alpha}$ has the following distribution:

$$
\begin{aligned}
P\left(z_{t}^{\alpha} \in A\right)=\int_{0}^{\infty} \frac{e^{-s^{2} / 2 t}}{\sqrt{2 \pi t}} \int_{\mathbb{R}} \frac{e^{-y^{2} / 2 s}}{\sqrt{2 \pi s}}( & \chi_{A}\left(\alpha^{1 / 4} e^{i \pi / 4} y\right) \\
& \left.+\chi_{A}\left(\alpha^{1 / 4} e^{-i \pi / 4} y\right)\right) d y d s
\end{aligned}
$$

$A$ being a Borel subset of the complex plane and $\chi_{A}$ being its characteristic function. Clearly the measure is concentrated on two rays of the complex plane $\alpha^{1 / 4} e^{i \pi / 4} \mathbb{R}$ and $\alpha^{1 / 4} e^{-i \pi / 4} \mathbb{R}$.

One can easily verify that random variable $z_{t}^{\alpha}$ has the following properties:

(i) $z_{t}^{\alpha} \sim t^{1 / 4} z_{1}^{\alpha}$,

(ii) $\mathbb{E}\left[\left(z_{t}^{\alpha}\right)^{k}\right]=0, k=1,2,3$,

(iii) $\mathbb{E}\left[\left(z_{t}^{\alpha}\right)^{4}\right]=-3 t$, 
(iv) $\mathbb{E}\left[\left|z_{t}^{\alpha}\right|^{2}\right]=2|\alpha t|^{1 / 2} \int_{0}^{\infty}\left(e^{-s^{2} / 2} / \sqrt{2 \pi}\right) s d s<+\infty$,

(v) $\mathbb{E}\left[e^{i \lambda z_{t}^{\alpha}}\right]=e^{-(t / 8) \alpha \lambda^{4}}$.

Moreover formula (27) can be written in Funaki's notation (see (6) and (7)). Indeed let us consider two independent Brownian motions $\{B(t)\}_{t \geq 0}$ and $\{w(t)\}_{t \geq 0}$ and define the process $\left\{X_{t}^{\alpha}\right\}_{t \geq 0}$ as

$$
X_{t}^{\alpha}:= \begin{cases}e^{i \pi / 4} \alpha^{1 / 4} B(w(t)) & \text { if } w(t) \geq 0 \\ e^{-i \pi / 4} \alpha^{1 / 4} B(-w(t)) & \text { if } w(t)<0 .\end{cases}
$$

Then (17) can then be written in the following form:

$$
e^{-\alpha(t / 8) \Delta^{2}} u_{0}(x)=\mathbb{E}\left[u_{0}\left(x+X_{t}^{\alpha}\right)\right]
$$

Remark 5. Analogous results can be obtained also in the case where $\Delta^{2}$ is replaced with higher powers of the Laplacian, namely, $\Delta^{4}, \Delta^{8}, \ldots, \Delta^{2^{n}}$. It is sufficient to iterate $n$-times formula (10). One obtains a formula with multiple Gaussian integrations, similar to the one proposed, for instance, in [17]. As in the Funaki approach, the probability measure of the complex random variable can also be obtained by composing three independent Brownian motions in a suitable way. In fact any even power of the Laplacian can be handled by means of the general formula (11), but a probabilistic interpretation in terms of the composition of several independent Brownian motions is not always possible. For instance, in the case where one considers $\Delta^{6}$, (11) gives the following result:

$$
e^{-\left(t / 2^{6}\right) \Delta^{6}} f(x)=\int_{-\infty}^{\infty} \widehat{g}_{t}(s) e^{i s(\Delta / 2)} u_{0}(x) d s,
$$

with

$$
\widehat{g}_{t}(s)=\frac{1}{2 \pi} \int_{-\infty}^{\infty} e^{-i s \xi} e^{-t \xi^{6}} d \xi
$$

We will not further develop these formulae here, but we will only focus on the case of $\Delta^{2}$.

\section{Feynman-Kac Type Formulae}

The process $\left\{X_{t}^{\alpha}\right\}_{t \geq 0}$ defined in (29) and appearing in (30) provides a probabilistic representation for the solution of (16) (under suitable analyticity assumptions on the initial datum $\left.u_{0}\right)$. On the other hand it has not independent increments, so it does not naturally give rise to generalizations of formula (30) to the case where (16) contains also a potential $V$, that is, to a Feynman-Kac formula of the form

$$
u(t, x)=\mathbb{E}\left[u_{0}\left(x+X_{t}^{\alpha}\right) e^{-\int_{0}^{t} V\left(x+X_{s}^{\alpha}\right) d s}\right]
$$

Indeed, by applying formally the Trotter product formula, one gets

$$
\begin{aligned}
& e^{-t\left(\left(\alpha \Delta^{2} / 8\right)+V\right)} u_{0}(x) \\
&=\lim _{n \rightarrow \infty}\left(e^{-(t / n)\left(\alpha \Delta^{2} / 8\right)} e^{-(t / n) V}\right)^{n} u_{0}(x) \\
&=\lim _{n \rightarrow \infty} \mathbb{E} {\left[e^{-(t / n) \sum_{k=1}^{n} V\left(x+\sum_{j=1}^{k} z_{j}^{\alpha}(t / n)\right)}\right.} \\
&\left.\times u_{0}\left(x+\sum_{j=1}^{n} z_{j}^{\alpha}\left(\frac{t}{n}\right)\right)\right],
\end{aligned}
$$

where $z_{j}^{\alpha}(t / n), j=1, \ldots n$, is a family of $n$ independent identically distributed random variables, distributed as $z_{t / n}^{\alpha}$ (see (28)). In the latter line one would be tempted to interpret the random variables $z_{j}^{\alpha}(t / n)$ as the independent increments of a complex-valued stochastic process $\left\{Z_{t}^{\alpha}\right\}_{t \geq 0}$, different from $\left\{X_{t}^{\alpha}\right\}_{t \geq 0}$, that is, to interpret the limit (34) as the cylindrical approximations of an integral of the following form:

$$
\begin{aligned}
\mathbb{E}\left[u_{0}\left(x+Z_{t}^{\alpha}\right) e^{-\int_{0}^{t} V\left(x+Z_{s}^{\alpha}\right) d s}\right] \\
=\lim _{n \rightarrow \infty} \mathbb{E}\left[e^{-(t / n) \sum_{k=1}^{n} V\left(x+\sum_{j=1}^{k} z_{j}^{\alpha}(t / n)\right)}\right. \\
\left.\times u_{0}\left(x+\sum_{j=1}^{n} z_{j}^{\alpha}\left(\frac{t}{n}\right)\right)\right] .
\end{aligned}
$$

In fact such a process cannot exist, as its construction would be possible providing the weak convergence of the sequence of complex random variables $\sum_{j=1}^{n} z_{j}^{\alpha}(t / n)$ as $n \rightarrow \infty$. By using the scaling properties of the random variable $z_{t}^{\alpha}$, this is equivalent to the weak convergence of the sequence $n^{-1 / 4} \sum_{j=1}^{n} z_{j}^{\alpha}(1)$. As $z_{j}^{\alpha}(1)$ are independent identically distributed complex random variables with finite covariance, then the sequence $n^{-1 / 2} \sum_{j=1}^{n} z_{j}^{\alpha}(1)$ has a Gaussian limit. Consequently, the sequence $n^{-1 / 4} \sum_{j=1}^{n} z_{j}^{\alpha}(1)$ cannot converge weakly, as erroneously stated in [25], and formula (35) cannot be interpreted as an integral with respect to the measure associated to a process but just as the limit (34). Indeed the present section is devoted to the proof, for a suitable class of continuous functions $V$ and initial datum $u_{0}$, of a Feynman-Kac formula representing the solution of the Cauchy problem (8) as the limit of a sequence of finite dimensional approximations.

The implementation of formula (35) presents some technical problems, which do not appear in the proof of the classical Feynman-Kac formula (for the heat equation with potential). The first one is the definition of the integrals involved. In fact, since the random variables $z^{\alpha}(t / n)$ are complex valued, the real valued function $V$ as well as the initial datum $u_{0}$ has to admit an analytic extension to an entire function on the complex plane. We cannot require that it is 
bounded on $\mathbb{C}$; otherwise we could consider only the trivial case. Consequently we will integrate unbounded function, and in principle the convergence of the integrals has to be checked. In fact, for a large class of potentials, the integrals are not absolutely convergent and have to be defined in a suitable way.

The second problem concerns the proof that the integral (35) represents the solution of the Cauchy problem (8). Even if the second line of (35) recalls Trotter's product, this formula cannot be directly applied since it does not hold for general $\alpha \in \mathbb{C}$ and $V$ continuous real valued function.

The problem of the proof of a Feynman-Kac type formula for equations of the form (8) has been analyzed in [22], where the case of a linear potential $V$ is handled, and in [39], but a detailed proof for a sufficiently large class of potentials $V$ is still lacking. We generalized these results to the case where $V$ is linear in the space variable and presents an explicit time dependence.

Theorem 6. Let $a: \mathbb{R} \rightarrow \mathbb{C}$ be a continuous function and let us denote $M:=\max _{s \in[0, t]}|a(s)|$. Let $u_{0}$ be of the form $u_{0}(x)$ $=\int_{\mathbb{R}} e^{i x y} d \mu_{0}(y)$, where $\mu_{0}$ is a complex bounded variation measure on $\mathbb{R}$ satisfying the following condition:

$$
\int_{\mathbb{R}} e^{(|\alpha| t / 8)(|y|+M t)^{4}} d\left|\mu_{0}\right|(y)<\infty
$$

Then the solution of the Cauchy problem:

$$
\begin{aligned}
\frac{\partial u}{\partial t}(x, t)=-\frac{\alpha}{8} & \frac{\partial^{4}}{\partial x^{4}} u(x, t)-i a(t) x u(t, x) \\
& -\infty<x<\infty, 0 \leq t<\infty \\
u(x, 0)= & u_{0}(x),
\end{aligned}
$$

is given by

$$
\begin{aligned}
& u(t, x)=\mathbb{E}\left[u_{0}\left(x+Z_{t}^{\alpha}\right) e^{-i \int_{0}^{t} a(t-s)\left(x+Z_{s}^{\alpha}\right) d s}\right] \\
&:=\lim _{n \rightarrow \infty} \mathbb{E}[ u_{0}\left(x+\sum_{j=1}^{n} z_{j}^{\alpha}\left(\frac{t}{n}\right)\right) \\
&\left.\times e^{-i t / n \sum_{k=1}^{n} a(t-(k t / n))\left(x+\sum_{j=1}^{k} z_{j}^{\alpha}(t / n)\right)}\right],
\end{aligned}
$$

Proof. Under the stated assumptions, the finite dimensional integrals appearing in formula (38) assume the following form:

$$
\begin{array}{r}
e^{-(i t x / n) \sum_{k=1}^{n} a(t-(k t / n))} \mathbb{E}\left[e^{-i(t / n) \sum_{k=1}^{n} a(t-(k t / n)) \sum_{j=1}^{k} z_{j}^{\alpha}(t / n)}\right. \\
\left.\times \int_{\mathbb{R}} e^{i x y} e^{i y \sum_{j=1}^{n} z_{j}^{\alpha}(t / n)} d \mu_{0}(y)\right],
\end{array}
$$

where $z_{j}^{\alpha}(t / n), j=1, \ldots n$, is a family of $n$ independent identically distributed random variables, distributed as $z_{t / n}^{\alpha}$.
Now by applying the Fubini theorem, which holds because of condition (36), the latter is equal to

$$
\begin{aligned}
& e^{-(i t x / n)} \sum_{k=1}^{n} a(t-(k t / n)) \\
& \times \int_{\mathbb{R}} e^{i x y} \mathbb{E}\left[e^{-i \sum_{j=1}^{n} z_{j}^{\alpha}(t / n)\left(-y+t / n \sum_{k=j}^{n} a(t-(k t / n))\right)}\right] d \mu_{0}(y) \\
&= e^{-(i t x / n) \sum_{k=1}^{n} a(t-(k t / n))} \\
& \times \int_{\mathbb{R}} e^{i x y} e^{-(\alpha / 8)(t / n) \sum_{j=1}^{n}\left(-y+t / n \sum_{k=j}^{n} a(t-(k t / n))\right)^{4}} d \mu_{0}(y) .
\end{aligned}
$$

By dominated convergence theorem, the limit as $n \rightarrow \infty$ of the last line is equal to

$$
e^{-i x \int_{0}^{t} a(s) d s} \int_{\mathbb{R}} e^{i x y} e^{-(\alpha / 8) \int_{0}^{t}\left(-y+\int_{s}^{t} a(t-u) d u\right)^{4} d s} d \mu_{0}(y),
$$

which is the solution of Cauchy problem (37).

\section{Conclusions}

In this paper we have proposed the construction of a particular probabilistic representation for the solution of the equation $\dot{u}=-\alpha \Delta^{2} u+V$ in terms of a Feynman-Kac type formula. The class of potentials $V$ which can be handled by requiring that the probabilistic integrals are defined in Lebesgue sense, that is, as absolutely convergent integrals, is rather restricted because of the complex nature of the process. A generalization of these results to more general potentials requires the implementation of an integration technique, in infinite dimensions, of a different type, by relaxing the absolute convergence of the integrals, as in the cases handled, for instance, in [6] concerning the functional integral representation for the solution of Schrödinger equations. This problem will be handled in a forthcoming paper.

\section{Acknowledgments}

Interesting discussions with Sergio Albeverio, Giuseppe Da Prato, Paolo Dai Pra, Franco Flandoli, Enrico Priola, and Luciano Tubaro are gratefully acknowledged, as well as the financial support of Fondazione Bruno Kessler, Trento, Italy.

\section{References}

[1] E. B. Dynkin, Theory of Markov Processes, Dover, Mineola, NY, USA, 2006.

[2] I. Karatzas and S. E. Shreve, Brownian Motion and Stochastic Calculus, Springer, New York, NY, USA, 1991.

[3] E. Sinestrari, "Accretive differential operators," Bollettino della Unione Matematica Italiana B, vol. 13, no. 1, pp. 19-31, 1976.

[4] S. Albeverio, "Wiener and feynman path integrals and their applications," Proceedings of Symposia in Applied Mathematics, vol. 52, pp. 163-194, 1997.

[5] S. Albeverio, R. Hoegh-Krohn, and S. Mazzucchi, Mathematical Theory of Feynman Path Integrals - An Introduction, vol. 523 of Lecture Notes in Mathematics, Springer, Berlin, Germany, 2nd edition, 2008. 
[6] S. Mazzucchi, Mathematical Feynman Path Integrals and Applications, World Scientific, Singapore, 2009.

[7] G. W. Johnson and M. L. Lapidus, The Feynman Integral and Feynman's Operational Calculus, Oxford University Press, New York, NY, USA, 2000.

[8] R. H. Cameron, "A family of integrals serving to connect the wiener and feynman integrals," Journal of Mathematical Physics, vol. 39, pp. 126-140, 1960.

[9] V. Y. Krylov, "Some properties of the distribution corresponding to the equation $\partial u / \partial t=(-1)^{q+1} \partial^{2 q} u / \partial x^{2 q}$," Doklady Akademii Nauk SSSR, vol. 132, pp. 1254-1257, 1960 (Russian), translated as Soviet Mathematics Doklady, vol. 1, pp. 760-763.

[10] K. Hochberg, "A signed measure on path space related to wiener measure," Annals of Probability, vol. 6, no. 3, pp. 433-458, 1978.

[11] K. Hochberg and E. Orsingher, "The arc-sine law and its analogs for processes governed by signed and complex measures," Stochastic Processes and Their Applications, vol. 52, no. 2, pp. 273-292, 1994.

[12] K. Hochberg, "Central limit theorem for signed distributions," Proceedings of the American Mathematical Society, vol. 79, no. 2, pp. 298-302, 1980.

[13] K. Nishioka, "Stochastic calculus for a class of evolution equations," Japanese Journal of Mathematics, vol. 11, no. 1, pp. 59-102, 1985.

[14] L. Beghin, K. J. Hochberg, and E. Orsingher, "Conditional maximal distributions of processes related to higher-order heattype equations," Stochastic Processes and Their Applications, vol. 85, no. 2, pp. 209-223, 2000.

[15] D. Levin and T. Lyons, "A signed measure on rough paths associated to a PDE of high order: results and conjectures," Revista Matematica Iberoamericana, vol. 25, no. 3, pp. 971-994, 2009.

[16] T. Funaki, "Probabilistic construction of the solution of some higher order parabolic differential equation," Proceedings of the Japan Academy A, vol. 55, no. 5, pp. 176-179, 1979.

[17] K. Hochberg and E. Orsingher, "Composition of stochastic processes governed by higher-order parabolic and hyperbolic equations," Journal of Theoretical Probability, vol. 9, no. 2, pp. 511-532, 1996.

[18] E. Orsingher and X. Zhao, "Iterated processes and their applications to higher order differential equations," Acta Mathematica Sinica, vol. 15, no. 2, pp. 173-180, 1999.

[19] S. Bochner, Harmonic Analysis and the Theory of Probability, University of California Press, Berkeley, Calif, USA, 1955.

[20] K. Burdzy, "Some path properties of iterated brownian motion," in Seminar on Stochastic Processes, vol. 33 of Progress in Probability, pp. 67-87, Birkhäuser, Boston, Mass, USA, 1993.

[21] H. Allouba, "Brownian-time processes: the PDE connection II and the corresponding feynman-kac formula," Transactions of the American Mathematical Society, vol. 354, no. 11, pp. 46274637, 2002.

[22] A. Madrecki and M. Rybaczuk, "New feynman-kac type formula," Reports on Mathematical Physics, vol. 32, no. 3, pp. 301327, 1993.

[23] K. Burdzy and A. Madrecki, "An asymptotically 4-stable process," in Proceedings of the the Conference in Honor of JeanPierre Kahane, pp. 97-117, Journal of Fourier Analysis and Applications, Orsay, France, June 1993.

[24] K. Burdzy and A. Madrecki, "Ito formula for an asymptotically 4-stable process," Annals of Applied Probability, vol. 6, no. 1, pp. 200-217, 1996.
[25] P. Sainty, "Construction of a complex-valued fractional brownian motion of order N," Journal of Mathematical Physics, vol. 33, no. 9, pp. 3126-3149, 1992.

[26] R. Léandre, "Stochastic analysis without probability: study of some basic tools," Journal of Pseudo-Differential Operators and Applications, vol. 1, no. 4, pp. 389-400, 2010.

[27] R. Léandre, "A path-integral approach to the Cameron-MartinMaruyama-Girsanov formula associated to a bilaplacian," Journal of Function Spaces and Applications, vol. 2012, Article 458738, 9 pages, 2012.

[28] T. Hida, H. H. Kuo, J. Potthoff, and L. Streit, White Noise, Kluwer Academic Publishers, Dordrecht, The Netherlands, 1995.

[29] R. Léandre, "Path integrals in noncommutative geometry," in Encyclopedia of Mathematical Physics, J. P. Francoise, G. Naber, and S. T. Tsou, Eds., pp. 8-12, Elsevier, Oxford, UK, 2006.

[30] R. Léandre, "Theory of distribution in the sense of connes-hida and feynman path integral on a manifold," Infinite Dimensional Analysis, Quantum Probability and Related Topics, vol. 6, no. 4, pp. 505-517, 2003.

[31] Yu. L. Daletsky and S. V. Fomin, Measures and Differential Equations in Infinitedimensional Space, Kluwer Academic Publishers, Dordrecht, The Netherlands, 1991.

[32] N. V. Smorodina and M. M. Faddeev, "Probabilistic representation of solutions for a class of evolution equations," Journal of Mathematical Sciences, vol. 176, no. 2, pp. 239-254, 2011.

[33] L. L. Helms, "Biharmonic functions and brownian motion," Journal of Applied Probability, vol. 4, pp. 130-136, 1967.

[34] L. R. Bragg and J. W. Dettman, "Related problems in partial differential equations," Bulletin of the American Mathematical Society, vol. 74, pp. 375-378, 1968.

[35] R. J. Griego and R. Hersh, "Random evolutions, Markov chains, and systems of partial differential equations," Proceedings of the National Academy of Sciences of the USA, vol. 62, pp. 305-308, 1969.

[36] R. Hersh, "Direct solution of general one-dimensional linear parabolic equation via an abstract plancherel formula," Proceedings of the National Academy of Sciences of the USA, vol. 63, pp. 648-654, 1969.

[37] R. Hersh, "Explicit solution of a class of higher-order abstract cauchy problems," Journal of Differential Equations, vol. 8, no. 3, pp. 570-579, 1970.

[38] R. Hersh, "The method of transmutations," in Partial Differential Equations and Related Topics, vol. 446 of Lecture Notes in Mathematics, pp. 264-282, Springer, Berlin, Germany, 1975.

[39] B. Gaveau and P. Sainty, "A path integral formula for certain fourth-order elliptic operators," Letters in Mathematical Physics, vol. 15, no. 4, pp. 345-350, 1988. 


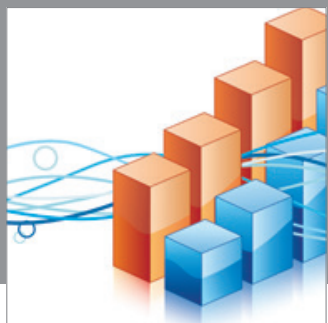

Advances in

Operations Research

mansans

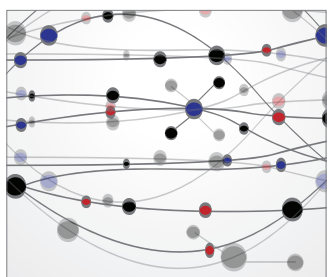

The Scientific World Journal
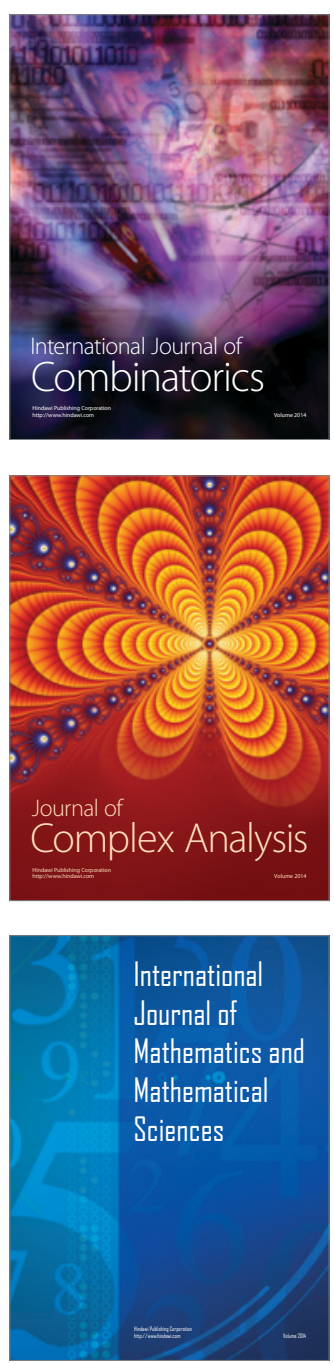
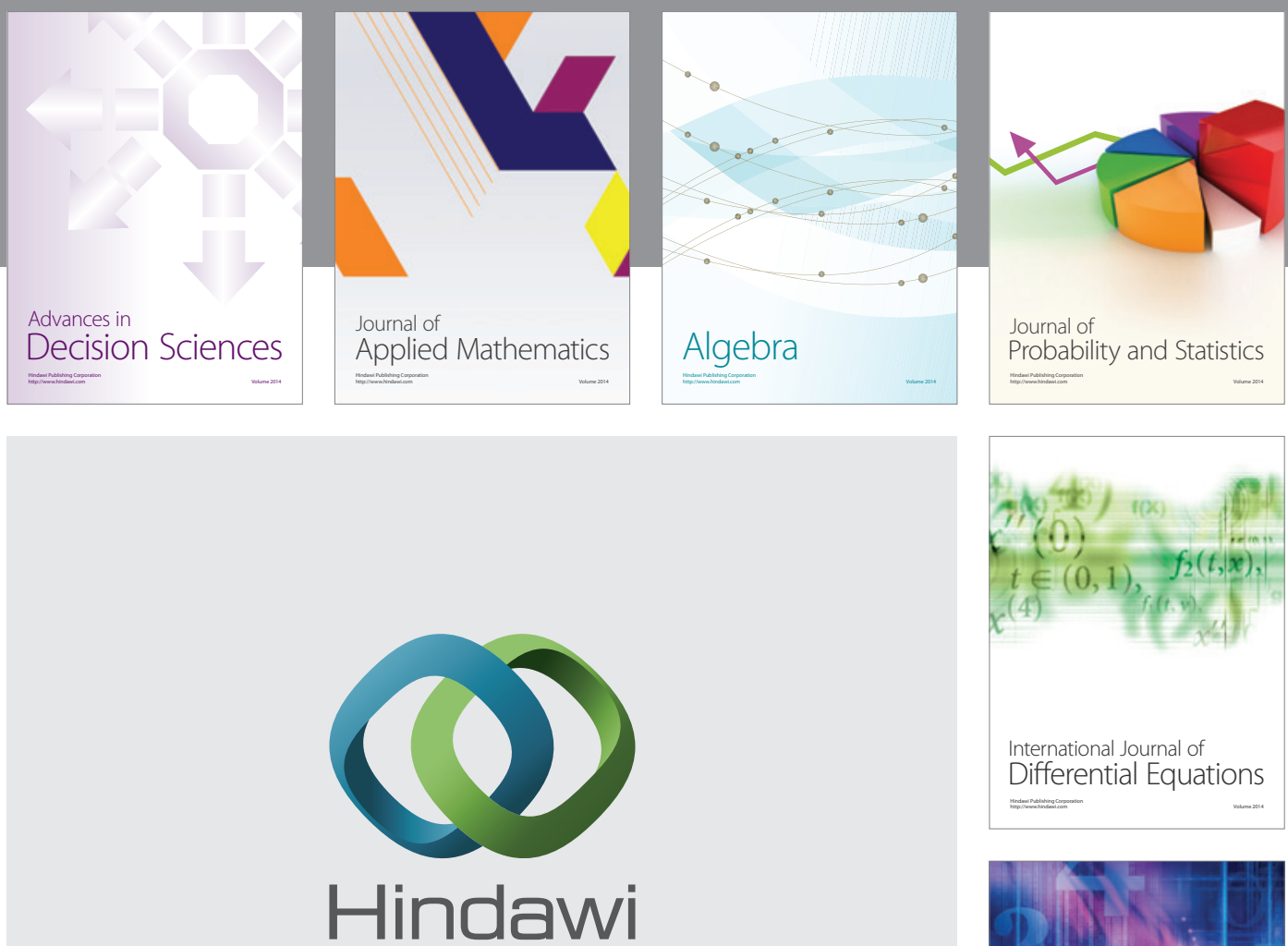

Submit your manuscripts at http://www.hindawi.com
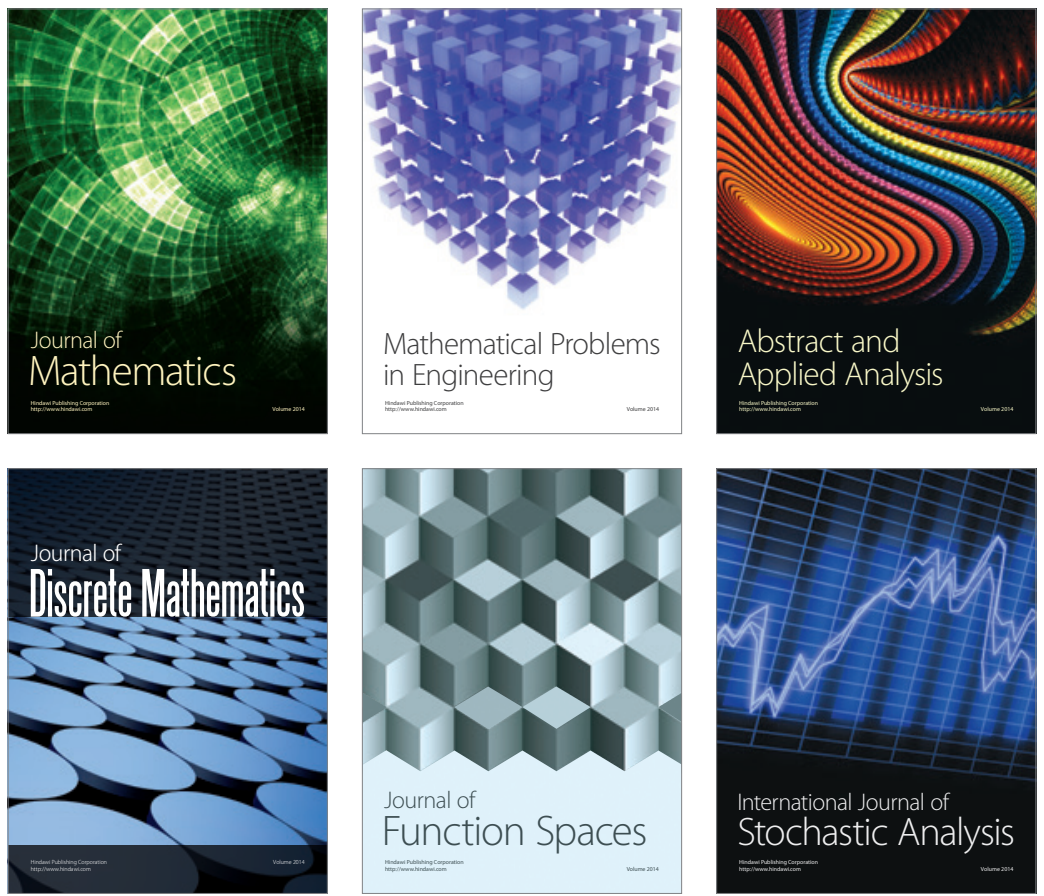

Journal of

Function Spaces

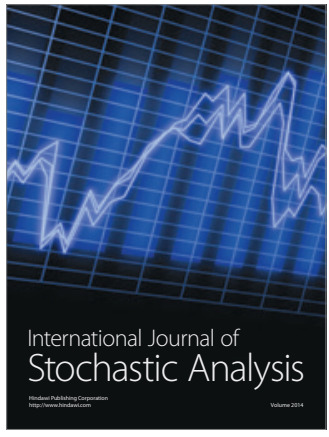

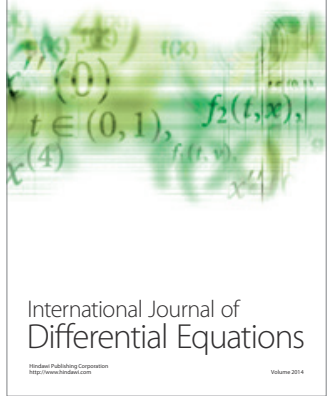
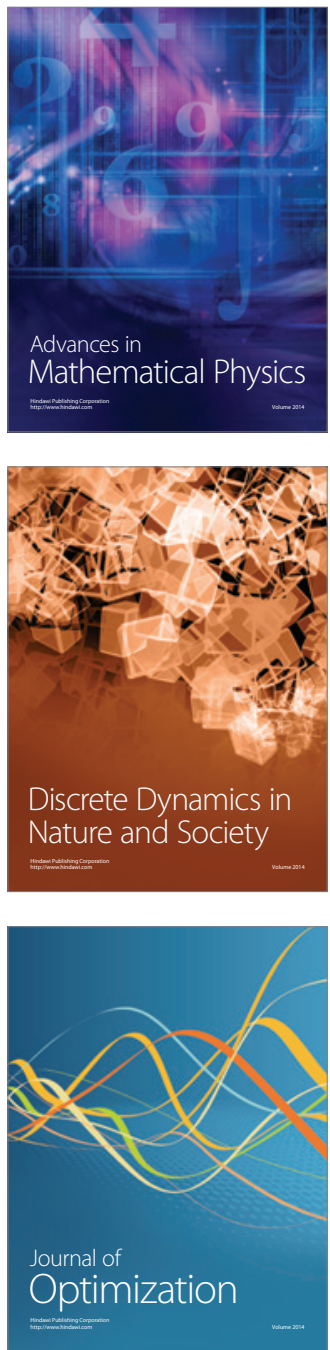